\title{
Echocardiographic indices of left ventricular hypertrophy and diastolic function in hypertensive patients with preserved LVEF classified as dippers and non-dippers
}

Monika Możdżan ${ }^{1,2}$, Karina Wierzbowska-Drabik'1, Małgorzata Kurpesa', Ewa Trzos', Tomasz Rechciński', Marlena Broncel², Jarosław D. Kasprzak ${ }^{1}$

${ }^{12 \text { nd }}$ Chair and Department of Cardiology, Medical University of Lodz, Poland 2Department of Internal Medicine and Clinical Pharmacology, Medical University of Lodz, Poland

Submitted: 15 May 2011

Accepted: 17 October 2011

Arch Med Sci 2013; 9, 2: 268-275

DOI: $10.5114 /$ aoms.2013.34534

Copyright $\odot 2013$ Termedia \& Banach

\section{Abstract}

Introduction: Long-lasting arterial hypertension causes left ventricular hypertrophy (LVH) and impairs left ventricular diastolic function. Our aim was to compare echocardiographic parameters between hypertensive patients defined as dippers and non-dippers during ambulatory blood pressure (BP) monitoring. Material and methods: We analysed 61 consecutive subjects with treated hypertension undergoing 24-h BP monitoring and transthoracic echocardiographic examination and included in the study patients with preserved left ventricular ejection fraction ( $E F \geq 50 \%$ ). Echocardiographic and arterial pressure parameters were compared between the group classified as dippers $(n=26,57 \pm 13$ years, 16 males) and non-dippers ( $n=35,60 \pm 12$ years, 24 males) according to present or absent decrease of BP during the night $>10 \%$. Echocardiographic data were compared between both groups and control subjects without hypertension.

Results: Dippers had lower average systolic, diastolic and mean arterial pressure during the night hours but did not differ according to the mean pressure calculated from a 24-hour period. All echocardiographic parameters were similar in dippers and non-dippers. All patients with arterial hypertension presented with larger dimension of both ventricles and left atrium, thicker left ventricular walls, higher LV mass and mass index and preserved EF and E/A ratio as compared with normotensive controls. Normal geometry, concentric remodelling and eccentric hypertrophy were similarly distributed in both groups. Concentric hypertrophy was more prevalent in non-dippers as compared to the dippers $(71.4 \%$ vs. $38.5 \%, p<0.043)$.

Conclusions: The concentric type of LVH is the prevalent pattern in non-dippers. Non-dipping blood pressure pattern may be responsible for the development of left ventricular concentric hypertrophy secondary to hypertension.

Key words: echocardiography, ambulatory blood pressure monitoring.

\section{Introduction}

Long-lasting, essential hypertension is usually associated with left ventricular hypertrophy (LVH) and impairs left ventricular diastolic function. Hypertension leads to different patterns of abnormal geometry of the left ventricle. The spectrum of cardiac morphological adaptation to high blood pressure encompasses concentric and eccentric hypertrophy. Eccentric

\author{
Corresponding aurthor: \\ Monika Możdżan MD \\ Department \\ of Internal Medicine \\ and Clinical Pharmacology \\ Medical University of Lodz \\ 1/5 Kniaziewicza St \\ 91-347 Lodz, Poland \\ Phone: +48 422516135 \\ Phone/fax: +48 426511059 \\ E-mail: \\ monika.modzan@umed.lodz.pl
}


hypertrophy is characterized by a spherical left ventricle, increased stroke volume and low peripheral vascular resistance. Contrarily, most patients with concentric hypertrophy have an elliptic left ventricle, normal stroke volume and high peripheral vascular resistance [1]. Although any type of LVH increases the incidence of cardiovascular disease, the concentric type of left ventricular hypertrophy has been identified as the cardiac structural parameter that is most strongly related with cardiovascular risk [2, 3]. Blood pressure profile lacking sufficient physiological decrease during the night is believed to especially promote structural changes in the left ventricle [4]. The renin-angiotensinaldosterone system (RAAS) is implicated in this process $[5,6]$. In advanced stages the remodelling process causes diastolic dysfunction and organ damage, and poses an elevated risk of cardiovascular events [7, 8]. Moreover, left ventricular mass index (LVMI) is a significant predictor of left atrial enlargement (LAE), which is associated with increased cardiovascular hospitalization and mortality risk [9].

Ambulatory blood pressure (BP) values have been shown to have a better relationship to the left ventricular mass (LVM) of hypertensive patients with $\mathrm{LVH}$ than conventional office BP measurements [10]. Similarly, the regression of $\mathrm{LVH}$ associated with improved cardiovascular prognosis may be more closely correlated with reductions in ambulatory BP than office blood pressure [11-14]. Day-time and night-time blood pressure values and their changes due to treatment are related to each other [15], but the prognostic value of night-time blood pressure has been found superior [16]. Moreover, patients with a non-dipper circadian pattern of BP are at higher risk of micro- and macrovascular complications, such as cerebrovascular and cardiovascular complications, than individuals with a dipper circadian rhythm $[17,18]$. Non-dipping of nocturnal BP seems to be a determinant of cardiac remodelling and LV diastolic dysfunction (LVDD) and may result in a cardiovascular risk independent of the increase in LVM in both hypertonic patients and in normotensives [19]. Twenty-four ambulatory blood pressure monitoring (24-ABPM) values of average systolic pressure, unlike office measurements, correlate with LVH indices in hypertensive subjects [20]. The data also suggest that early structural cardiac changes such as an increase in septal thickness and a decrease in LV ejection time are related to ambulatory blood pressure profile [21]. However, there are conflicting data regarding the relationship between the circadian rhythm of BP, especially non-dipping nocturnal BP, and LVH and left ventricular diastolic function in patients with essential hypertension.

We hypothesized that patients with the non-dipper pattern would have a more pronounced left ventricular hypertrophy and diastolic dysfunction than patients with the dipper pattern. Accordingly, we evaluated and compared structural echocardiographic parameters in hypertensive patients known to have dipper and non-dipper characteristics during 24-h BP monitoring.

\section{Material and methods}

\section{Study group}

Data were collected from the medical records of 76 treated patients with essential hypertension who underwent ambulatory BP monitoring and transthoracic echocardiographic examination at the Department of Cardiology during a period of 14 months (from April 2009 to June 2010). Treatment was individualized and based on lifestyle and pharmacological measures. Diuretics (43\%), $\beta$-blockers (34\%), angiotensin-converting enzyme inhibitors (ACEI $86 \%$ ), angiotensin receptor blockers (ARB - 26\%) and calcium channel blockers (38\%), alone or combined, were the antihypertensive drugs most frequently used.

Hypertensive subjects can be divided into 2 groups, dippers and non-dippers, according to the presence or absence of a nocturnal fall of blood pressure of more than $10 \%$, measured as mean arterial pressure (MAP). The MAP was defined as $M A P=2 / 3 \times D B P+1 / 3 \times S B P$.

Inclusion criteria were well-controlled hypertension with preserved left ventricular ejection fraction (EF $\geq 50 \%$ ) and exclusion criteria were: severe hypertension (systolic BP (SBP)] $\geq 210 \mathrm{~mm} \mathrm{Hg}$ and/or diastolic BP (DBP) $\geq 115 \mathrm{~mm} \mathrm{Hg}$ ), secondary hypertension, significant kidney disease, valvular heart disease, heart failure and history of ischaemic heart disease. Among the patients with preserved left ventricular ejection fraction ( $E F \geq 50 \%$ ), 35 nondippers (60 \pm 12 years, 24 males) and 26 dippers (57 \pm 13 years, 16 males) matched for age, sex, and body mass index were enrolled in this study. The control group consisted of 25 healthy individuals (53 \pm 12 years, 13 males). Echocardiographic and arterial pressure parameters were compared between dippers and non-dippers.

\section{Ambulatory blood pressure monitoring}

Ambulatory BP was recorded using an oscillometric device (Healthcare CardioNavigator V 2.601), and measurements were automatically taken every 15 min throughout 16 h during the day (07.00$23.00 \mathrm{~h}$ ) and at 20-minute intervals during the night (23.00-7.00 h). Each ABPM dataset was first automatically scanned to remove artefactual readings, according to preselected editing criteria. Systolic readings $>260 \mathrm{~mm} \mathrm{Hg}$ or $<70 \mathrm{~mm} \mathrm{Hg}$ and diastolic readings $>150 \mathrm{~mm} \mathrm{Hg}$ or $<40 \mathrm{~mm} \mathrm{Hg}$ were automatically discarded. The recording was then ana- 
lysed to obtain $24 \mathrm{~h}$, day-time and night-time average SBP, DBP and heart rates.

\section{Echocardiography}

Transthoracic echocardiographic examination was performed with Vivid 7 GE with measurements of chamber dimensions taken from 2D mode, mitral inflow and calculation of LV mass and relative wall thickness (RWT). Linear measurements were made according to the European Society of Echocardiography [22]. Left ventricle volumes used to estimate ejection fraction (EF) were determined using the modified biplane Simpson's method. The protocol included assessment of mitral inflow pattern (in apical four-chamber view with the pulse Doppler sample volume at the tips of opened mitral valve leaflets). All echocardiographic measurements were performed by a cardiologist with subspecialty training in echocardiography. For LV mass the coefficient of variability for intra-observed repeated measures was less than $5 \%$.

Relative wall thickness (RWT) was defined as [23]: RWT $=2 \times$ LVPWd/LVDd, where: LVPWd - posterior wall diastolic thickness, LVDd - left ventricular diastolic diameter. LV mass was calculated according to the Devereux formula: LV mass (g) = $1.04\left((\text { LVDd + IVSd + LVPWd })^{3}-\right.$ LVDd $\left.^{3}\right)-13.6$ where: LVDd - left ventricular diastolic diameter, IVSd - intraventricular septal diameter, LVPWd posterior wall diastolic thickness. The left ventricular mass index (LVMI) was obtained as an indicator of LVH by echocardiography as a ratio of LVM and body surface area.

LV hypertrophy (LVH) was defined by LVMI thresholds of $125 \mathrm{~g} / \mathrm{m}^{2}$ for men, and $110 \mathrm{~g} / \mathrm{m}^{2}$ for women. Left ventricular relative thickness and left ventricular mass defined left ventricular geometric patterns - normal geometry, concentric remodelling, eccentric hypertrophy and concentric hypertrophy. Concentric LV hypertrophy - RWT $\geq 0.42$ with an increased LVMI; eccentric LV hypertrophy increased LVMI with an RWT < 0.42; concentric remodelling - RWT $\geq 0.42$ with a normal LVMI.
Diastolic function was assessed by recording mitral flow with standard pulsed Doppler technique, and measurements of early diastolic peak flow velocity $(E)$, late diastolic peak flow velocity $(A)$ and the ratio of early to late flow velocity peaks (E/A ratio).

We accepted E/A ratio $<1$ as an indicator of delayed relaxation profile; $E / A>2$ as typical for restriction; and values of E/A between 1 and 2 as either normal (or) pseudonormal pattern. Since the deceleration time of early waves shows a strong inverse correlation with left ventricular filling pressure, well documented with systolic dysfunction, we used the deceleration time below 150 ms as a simplified criterion of pseudonormalization. Pseudonormal pattern was also recognised with patients with a prolonged difference between duration of atrial reversal flow in the pulmonary vein and mitral flow in atrial phase, delta At $\geq 20 \mathrm{~ms}[24,25]$.

\section{Statistical analysis}

Values are expressed as means \pm standard deviations (SD) or as percentages. Differences between groups were assessed by analysis of variance (ANOVA). Mean values for dipper and non-dipper patients were compared using Student's $t$-test for independent samples. $\chi^{2}$ test was used to compare categorical variables between groups. Correlations were obtained by using Pearson's linear regression model. Values of $p$ below 0.05 were considered statistically significant. Data management and statistical analysis were performed using Med-Calc V. 6.14. (Frank Schoonjans, Belgium).

\section{Results}

Demographic and clinical characteristics of the study population are reported in Table I with no significant differences between the studied groups.

By definition, non-dippers showed higher average systolic and diastolic pressure during the night, although 24-hour blood pressure was similar between the groups (Table II).

Table I. Clinical characteristics of study population

\begin{tabular}{|lccc|}
\hline Parameter & Dipper $(n=26)$ & Non-dipper $(n=35)$ & Statistical significance \\
\hline Age [years] & $57 \pm 13$ & $60 \pm 12$ & NS \\
\hline Sex $(\mathrm{M} / \mathrm{F})$ & $16 / 10$ & $24 / 11$ & NS \\
\hline BMI $\left[\mathrm{kg} / \mathrm{m}^{2}\right]$ & $27.8 \pm 5.4$ & $29.6 \pm 4.4$ & NS \\
\hline Normal weight/overweight/obese [\%] & $35 / 38 / 27$ & $20 / 43 / 37$ & NS \\
\hline Duration of hypertension [months] & $54(6-192)$ & $43(10-216)$ & NS \\
\hline Daytime SBP [mm Hg] & $123.15 \pm 12.9$ & $118.04 \pm 11.2$ & NS \\
\hline Daytime DBP $[\mathrm{mm} \mathrm{Hg}]$ & $71.3 \pm 9.6$ & $67.6 \pm 9.1$ & NS \\
\hline
\end{tabular}

$B M I$ - body mass index, BMI 19-24.99 kg/m² - normal weight, BMI $25-29.99 \mathrm{~kg} / \mathrm{m}^{2}$ - overweight, $B M \mathrm{l}>30 \mathrm{~kg} / \mathrm{m}^{2}$ - obese, DBP-diastolic blood pressure, SBP - systolic blood pressure 
Expectedly, in comparison with the normotensive group, patients with hypertension displayed larger ventricles and left atrium, thicker left ventricular walls and higher LV mass and mass index and lower mean EF and E/A ratio (Table III).

No statistically significant differences were found between dippers and non-dippers as regards ejection fraction, chamber dimensions, wall thickness, left ventricular mass and mass index and E/A ratio; i.e. the 2 groups did not significantly differ in left atrial diameter, end-diastolic diameter, end-systolic diameter, interventricular wall thickness, or posterior wall thickness (Table III).

According to echocardiographic measurements, 37 of the 61 patients $(60.7 \%)$ were found to have LVH (left ventricular mass index LVMI $>125 \mathrm{~g} / \mathrm{m}^{2}$ in men and $>110 \mathrm{~g} / \mathrm{m}^{2}$ in women). LVH was concentric in 35 patients and eccentric in the remaining 2 patients (Table IV). There was a statistically significant difference in LV geometric pattern distri-
Table II. Comparison of 24-h BP data. ANOVA test with Tukey's multiple comparison post-test was used

\begin{tabular}{|lccc|}
\hline Parameter & $\begin{array}{c}\text { Group A } \\
\text { dippers } \\
(n=26)\end{array}$ & $\begin{array}{c}\text { Group B } \\
\text { non-dippers } \\
(n=35)\end{array}$ & Value of $p$ \\
\hline $\begin{array}{l}\text { Night-time SBP } \\
{[\mathrm{mm} \mathrm{Hg}]}\end{array}$ & $103 \pm 13$ & $114 \pm 13$ & $<0.001$ \\
\hline $\begin{array}{l}\text { Night-time DBP } \\
\text { [mm Hg] }\end{array}$ & $56 \pm 9$ & $61 \pm 10$ & 0.022 \\
\hline $\begin{array}{l}\text { Night-time MABP } \\
\text { [mm Hg] }\end{array}$ & $72 \pm 10$ & $80 \pm 11$ & 0.004 \\
\hline $\begin{array}{l}\text { 24-hour SBP } \\
\text { [mm Hg] }\end{array}$ & $118 \pm 13$ & $117 \pm 11$ & NS \\
\hline $\begin{array}{l}\text { 24-hour DBP } \\
\text { [mm Hg] }\end{array}$ & $67 \pm 9$ & $66 \pm 9$ & NS \\
\hline $\begin{array}{l}\text { 24-hour MABP } \\
\text { [mm Hg] }\end{array}$ & $85 \pm 10$ & $83 \pm 9$ & NS \\
\hline
\end{tabular}

$S B P$ - systolic blood pressure, DBP - diastolic blood pressure, $M A B P-$ mean arterial blood pressure $(M A B P=2 / 3 \times D B P+1 / 3 \times S B P)$

Table III. Comparison of echocardiographic data. ANOVA test with Tukey's multiple comparison post-test was used

\begin{tabular}{|c|c|c|c|c|c|c|c|}
\hline Parameter & $\begin{array}{l}\text { Group A } \\
\text { dippers } \\
(n=26)\end{array}$ & $\begin{array}{c}\text { Group B } \\
\text { non-dippers } \\
(n=35)\end{array}$ & $\begin{array}{l}\text { Group C } \\
\text { controls } \\
(n=25)\end{array}$ & Value of $p$ & $\begin{array}{c}\text { Value of } p \\
\text { A vs. B }\end{array}$ & $\begin{array}{c}\text { Value of } p \\
\text { A vs. C }\end{array}$ & $\begin{array}{c}\text { Value of } p \\
\text { B vs. C }\end{array}$ \\
\hline LVd [mm] & $47 \pm 4$ & $49 \pm 4$ & $44 \pm 5$ & 0.004 & NS & NS & $<0.01$ \\
\hline LVs [mm] & $32 \pm 4$ & $35 \pm 3$ & $33 \pm 5$ & NS & NS & NS & NS \\
\hline LV PWd [mm] & $11 \pm 2$ & $12 \pm 1$ & $11 \pm 1$ & 0.016 & NS & NS & $<0.05$ \\
\hline LV PWs [mm] & $15 \pm 2$ & $15 \pm 1$ & $13 \pm 1$ & $<0.0001$ & NS & $<0.01$ & $<0.001$ \\
\hline LV IVSd [mm] & $12 \pm 2$ & $13 \pm 2$ & $11 \pm 1$ & 0.01 & NS & NS & $<0.01$ \\
\hline LV IVSs [mm] & $15 \pm 2$ & $16 \pm 2$ & $12 \pm 2$ & 0.0002 & NS & $<0.01$ & $<0.001$ \\
\hline $\mathrm{LA}[\mathrm{mm}]$ & $41 \pm 6$ & $43 \pm 6$ & $36 \pm 3$ & 0.0004 & NS & $<0.05$ & $<0.001$ \\
\hline $\mathrm{RV}[\mathrm{mm}]$ & $26 \pm 2$ & $27 \pm 3$ & $23 \pm 2$ & $<0.0001$ & NS & $<0.001$ & $<0.001$ \\
\hline EF [\%] & $59 \pm 5$ & $55 \pm 5$ & $62 \pm 3$ & 0.0002 & NS & NS & $<0.001$ \\
\hline$E / A$ & $1.0 \pm 0.3$ & $0.8 \pm 0.3$ & $1.4 \pm 0.5$ & $<0.001$ & NS & $<0.05$ & $<0.001$ \\
\hline LV mass [g] & $241 \pm 62$ & $291 \pm 67$ & $158 \pm 40$ & $<0.001$ & NS & $<0.001$ & $<0.001$ \\
\hline $\begin{array}{l}\text { LV mass index } \\
{\left[\mathrm{g} / \mathrm{m}^{2}\right]}\end{array}$ & $125 \pm 36$ & $142 \pm 31$ & $94 \pm 17$ & $<0.001$ & NS & $<0.001$ & $<0.001$ \\
\hline $\begin{array}{l}\text { RWT (relative } \\
\text { wall thickness) }\end{array}$ & $49 \pm 8$ & $50 \pm 6$ & $47 \pm 11$ & NS & NS & NS & NS \\
\hline
\end{tabular}

LVd - left ventricular diastolic diameter, LVs - left ventricular systolic diameter, LV PWd-posterior wall diastolic thickness, LV PWs - posterior wall systolic thickness, LV IVSd - interventricular septum thickness in diastole, LV IVSs - interventricular septum thickness in systole, LA - left atrial diameter, $R V$ - right ventricular diameter, $E F$ - left ventricle ejection fraction, $E / A$ - transmitral $E$ wave velocity/transmitral $A$ wave velocity ratio, $L V$ mass - left ventricular mass, RWT - relative wall thickness

Table IV. Comparison of left ventricular geometric patterns between dipper and non-dipper groups $\left(\chi^{2}\right.$ test was used for comparison of proportions)

\begin{tabular}{|lcccc|}
\hline Group & Normal LV geometry & Concentric remodelling & Concentric hypertrophy & Eccentric hypertrophy \\
\hline $\begin{array}{l}\text { Dippers } \\
n=26 ; 100 \%\end{array}$ & $3(11.5 \%)$ & $11(42.3 \%)$ & $10(38.5 \%)$ & $2(7.6 \%)$ \\
\hline $\begin{array}{l}\text { Non-dippers } \\
n=35,100 \%\end{array}$ & $3(8.6 \%)$ & $7(20 \%)$ & $25(71.4 \%)^{*}$ & $0(0 \%)$ \\
$*_{p}<0.043$ & & & & \\
\hline
\end{tabular}


bution between the two groups $(p=0.043)$. This was due to the increased prevalence of concentric hypertrophy in non-dippers compared to dippers (71.4\% vs. $38.5 \%)$.

\section{Discussion}

We studied the relationship between left ventricular mass (LVM) and $24 \mathrm{~h}$ ambulatory blood pressure monitoring (ABPM) in 61 patients with treated, long-standing, well-controlled hypertension.

The main finding of our study is increased prevalence of concentric LVH in the night-time non-dipper group. It has also been suggested that non-dipping could be responsible for the development of left ventricular hypertrophy. This was in disagreement with other studies which have addressed this issue. In the study by Cuspidi et al. [26] in a selected population of 111 subjects with a recent diagnosis of hypertension no differences were found in the correlation of daytime and night-time SBP and DBP with left ventricular mass and carotid wall thickness. Also in another study published by Cuspidi et al. [27] no differences in cardiac structure, analysed as a continuous variable, as well as prevalence of LVH, were found in relationship to dipping or non-dipping status in the treated essential hypertensives with or without BP control. In this group the extent of nocturnal BP decrease was not associated with LV mass or LVH prevalence. Therefore, the non-dipping profile did not identify hypertensive patients with greater cardiac damage. Cuspidi et al. concluded that a blunted reduction in nighttime blood pressure does not play a major role in the development of cardiovascular changes during the early phase of essential hypertension. The association between diurnal BP profile and target organ damage remains controversial. On the other hand, in a previous study by Cuspidi et al. [28] they suggested that the eccentric type of LVH, not the concentric, was the more frequent pattern in chronically treated patients. Moreover, the persistence of $\mathrm{LVH}$ was significantly dependent on BP levels achieved during treatment; indeed the prevalence of LVH was very low in patients with optimal BP control, whereas it was higher (37\%) in uncontrolled cases. Similarly to our results, Balci et al. [4] found that ventricular hypertrophy was higher in the nondipper group compared to the dipper group (42.9\% vs. $6.3 \%, p<0.03$ ), yet it was eccentric, not concentric. In addition, the findings of Cuspidi et al. [29] and Stenehjem et al. [30] suggest that the contribution of a blunted reduction in nocturnal BP to enlarged LV mass is significant and may play a pivotal role in the development of $\mathrm{LVH}$, during the early phase of essential hypertension. Moreover, subjects in whom the nocturnal decrease in blood pressure is blunted (non-dippers) have been report- ed to have a greater prevalence of organ damage and a less favourable outcome. A blunted fall in nocturnal BP also reflects the high level of cardiovascular risk in these patients. Nevertheless, in some studies the prognostic value of this phenomenon was lost when multivariate analysis included 24-h average blood pressure [3, 11, 12]. In agreement with our results, Felicio et al. [31] suggest that higher nocturnal systolic BP (NSBP) levels might be responsible for an increased prevalence of LVH in hypertensive patients with type 2 diabetes. However, in another study which enrolled diabetic patients, echocardiographic structural alterations correlated more strongly with systolic BP means than with non-dipper/dipper BP ratio [32].

Cicconetti et al. [33] selected 23 dippers and 17 non-dippers affected by newly diagnosed, never treated hypertension, who underwent 24-h ABPM and $M$-mode echocardiography. They found no significant differences between these 2 groups as regards the echocardiographic left ventricular and atrial dimensions or regarding the left ventricular mass, left ventricular mass index, or relative wall thickness. We obtained similar results. In our study all echocardiographic parameters - both ventricles and left atrium, left ventricular walls, LV mass, LV mass index and E/A ratio - were similar in dippers and non-dippers. In addition, Cicconetti et al. found no significant differences in the rate of either left ventricular remodelling or left ventricular hypertrophy. They suggested that in the early phases of hypertension, non-dipping status was not associated with a higher level of cardiac involvement as compared to dipping status. In contrast we found significant differences in the rate of left ventricular hypertrophy between groups.

The prevalence of left ventricular hypertrophy (LVH) has been mostly documented in populations of patients with essential hypertension [34], and selected hypertensive cohorts such as children. Left ventricular hypertrophy is common in children with newly diagnosed hypertension. As systolic ABP variables increase, there is greater likelihood for increased LVMI. Although the risk for LVH is associated with increased SBP, those patients with eccentric LVH had significantly higher DBP [35]. Brady et al. [36] even suggested that the initial examination of these children should include echocardiography, because neither the severity of casual BP elevation nor the presence of abnormal ambulatory BP results at initial diagnosis are predictive of $\mathrm{LVH}$.

It was concluded that LVH was related mainly to continuous systolic hypertension, and that LV diastolic dysfunction was related mainly to continuous diastolic hypertension. Therefore, it was suggested that LVH and LV diastolic dysfunction in hypertensive patients are caused by different mechanisms. 
Nevertheless, we did not observe a significant correlation either between LVH and high systolic pressure, or between LV diastolic dysfunction and high diastolic pressure.

Grossman et al. [37] also reported that in 60 patients with essential hypertension LVM was poorly related to both casual and exercise blood pressure, but was related to ABPM. They considered that blood pressure load is the best determinant of LVH. After evaluation of almost 150 hypertensives, Pascual et al. [38] found that the principal factor related to the presence of LVH is the value of systolic blood pressure throughout a $24-\mathrm{h}$ period. Feola et al. [39], in their study on untreated patients with essential hypertension, obtained similar results where they found that systolic ambulatory BP was significantly correlated with LVMI, while diastolic BP was not.

Numerous studies have addressed the predictive value for cardiovascular risk of the night-time BP as documented by ambulatory monitoring [40]. There are several mechanisms that could be responsible for a lower fall of BP during sleep [41]. The relationship between BMI and non-dipping was analysed. Cuspidi et al. [42] suggested that hypertensive patients with overweight had a reduced nocturnal BP fall and greater cardiac organ damage as compared with their lean counterparts, despite a similar overall BP load. In their study the prevalence of dipper pattern was 15\% lower in the overweight group as a whole, with a $17 \%$ difference in men and $13 \%$ in women. The prevalence of left ventricular hypertrophy was higher in patients with overweight, too. Nevertheless, in the study of Chamontin et al. [43], the non-dipper group showed a higher diurnal and nocturnal pulse pressure and a greater left ventricular mass index, in spite of their body mass index being almost identical to that of the dipper group. In our study mean body mass index was similar between dipper and non-dipper groups ( $27.8 \pm 5.4$ vs. $27.9 \pm 4.6)$. Despite the similar mean value, in the non-dipper group there were more patients whose BMI was above the reference values, with $37.14 \%$ obese and $42.86 \%$ overweight individuals vs. $26.92 \%$ obese and $38.46 \%$ overweight patients in the dipper group.

In our study we found that hypertensive patients compared to controls presented larger dimensions of both ventricles and left atrium, thicker left ventricular walls, and higher LV mass and mass index. These findings occurred independently of sex, age, $\mathrm{BMI}$ and diurnal BP levels. We also found impaired diastolic function in the hypertensive group of patients. Our data are in agreement with the study of di Bello et al. [44]. They compared pre-hypertensive and hypertensive patients with subjects without hypertension and found a higher LVMI in the first two groups. Moreover, they evaluated LV diastolic function using Doppler mitral flow velocity and PW-TDI recording at the mitral annulus level. They found that in hypertensive groups mild diastolic dysfunction was associated with systolic pressure load, and cardiac remodelling.

In the present work, the non-dipper group had a greater prevalence of concentric left ventricle hypertrophy, while concentric remodelling was not significantly higher in the dipper group. We have concluded that non-dipping seems to be responsible for the increased prevalence of concentric LVH in long-standing hypertension. We have suggested that the contribution of a blunted reduction in nocturnal BP to LVH is significant. It could be possible that the conversion from non-dipping to dipping pattern during treatment may play a role in the regression of myocardial hypertrophy.

It is our opinion that ABPM should be encouraged in the diagnosis and treatment of all hypertensive subjects as it gives information about the extent of night-time decrease, which could be a possible explanation for the high prevalence of LVH.

Our study included subjects with different onset, severity, and treatment modalities of hypertension. The small number of subjects may have influenced our results, such as the absence of statistical significance concerning prevalence of LV concentric remodelling in both studied groups. All study measurements were performed on patients under antihypertensive medications.

Additionally, our results are based only on 2Dmode echocardiographic technique and measurements. Although accepted for clinical investigation, this method is inferior to reference 3D echocardiographic or magnetic resonance based measurements of left ventricular mass.

In conclusion, the concentric type of LVH is the prevalent pattern in non-dippers. Non-dipping blood pressure pattern may be responsible for the development of left ventricular concentric hypertrophy secondary to hypertension.

\section{Acknowledgments}

The authors do not report any conflict of interest regarding this work.

\section{References}

1. Dávila DF, Donis JH, Odreman R, Gonzalez M, Landaeta A. Patterns of left ventricular hypertrophy in essential hypertension: should echocardiography guide the pharmacological treatment? Int J Cardiol 2008; 124: 134-8.

2. Muiesan ML, Salvetti M, Monteduro C, et al. Left ventricular concentric geometry during treatment adversely affects cardiovascular prognosis in hypertensive patients. Hypertension 2004; 43: 731-8.

3. Mancia G, De Backer G, Dominiczak A, Cifkova R, Fagard R, Germano G. 2007 ESC and ESH Guidelines for the man- 
agement of arterial hypertension. Eur Heart J 2007; 28: $1462-536$.

4. Balci B, Yilmaz O, Yesildag O. The influence of ambulatory blood pressure profile on left ventricular geometry. Echocardiography 2004; 21: 7-10.

5. Kieć-Wilk B, Stolarz-Skrzypek K, Sliwa A, Zdzienicka A, Kawecka-Jaszcz K. Peripheral blood concentrations of TGFbeta1, IGF-1 and bFGF and remodelling of the left ventricle and blood vessels in hypertensive patients. Kardiol Pol 2010; 68: 996-1002.

6. Matsumura K, Fujii K, Oniki H, Oka M, lida M. Role of aldosterone in left ventricular hypertrophy in hypertension. Am J Hypertens 2006; 19: 13-8.

7. Ciardullo AV, Azzolini L, Bevini M, et al. A diagnosis of left ventricular hypertrophy on ECG is associated with a highcardiovascular risk: findings from a 40- to 69-yearold cohort in general practice. Fam Pract 2004; 21: 63-5.

8. Koren MJ, Devereux RB, Casale PN. Relation of the left ventricular mass and geometry to morbidity an mortality in uncomplicated essential hypertension. Ann Intern Med 1991; 114: 345-52.

9. Tedesco MA, Di Salvo G, Ratti G, Natale F, larussi D, lacono A. Left atrial size in 164 hypertensive patients: an echocardiographic and ambulatory blood pressure study. Clin Cardiol 2001; 24: 603-7.

10. Roland A, Garcia-Puig J, Gosse P, et al. Ambulatory blood pressure in hypertensive patients with left ventricular hypertrophy: efficacy of first-line combination perindopril/ indapamide therapy. Vasc Health Risk Manag 2007; 3: 371-80.

11. Verdecchia P, Porcellati C, Schillaci G, et al. Ambulatory blood pressure. An independent predictor of prognosis in essential hypertension. Hypertension 1994; 24: 793-801.

12. Staessen JA, Thijs L, Fagard R, et al. Predicting cardiovascular risk using conventional vs ambulatory blood pressure in older patients with systolic hypertension. JAMA 1999; 282: 539-46.

13. Floriańczyk T, Werner B. Usefulness of ambulatory blood pressure monitoring in diagnosis of arterial hypertension in children and adolescents. Kardiol Pol 2008; 66: 12-8.

14. Wei TM, Lu LC, Ye XL, et al. Difference in blood pressure between supine and sitting positions in diabetic and nondiabetic subjects. Med Sci Monit 2009; 15: 123-7.

15. Mancia G, Parati G, Bilo G, et al. Assessment of long-term antihypertensive treatment by clinic an ambulatory blood pressure. Data from the ELSA Study. J Hypertens 2007; 25: 1087-94.

16. Sega R, Facchetti R, Bombelli M, et al. Prognostic value of ambulatory and home blood pressure compared with office blood pressure in the general population: follow-up results from the PAMELA study. Circulation 2005; 111: 1777-83.

17. Bouhanick B, Chamontin B. Should pulse pressure and day/night variations in blood pressure be seen as in dependent risk factors requiring correction or simply as markers to be taken into account when evaluating overal vascular risk? Diabetes Metab 2007; 33: 321-30.

18. Ukkola O, Vasunta RL, Kesäniemi YA. Non-dipping pattern in ambulatory blood pressure monitoring is associated with metabolic abnormalities in a random sample of middle-aged subjects. Hypertens Res 2009; 32: 1022-7.

19. Soylu A, Duzenli MA, Yazici M, Ozdemir K, Tokac M, Gok H. The effect of nondipping blood pressure patterns on cardiac structural changes and left ventricular diastolic functions in normotensives. Echocardiography 2009; 26: 378-87.

20. Dzau VJ, Antman EM, Black HR, et al. The cardiovascular disease continuum validated: clinical evidence of im- proved patient outcomes: part I: pathophysiology and clinical trial evidence (risk factors through stable coronary artery disease). Circulation 2006; 114: 2850-70.

21. Lemne C, Lindvall K, Georgiades A, Fredrikson M, de Faire U. Structural cardiac changes in relation to 24-h ambulatory blood pressure levels in borderline hypertension. J Intern Med 1995; 238: 49-57.

22. Lang RM, Bierig M, Devereux RB, et al. American Society of Echocardiography's Nomenclature and Standards Committee; Task Force on Chamber Quantification; American College of Cardiology Echocardiography Committee; American Heart Association; European Association of Echocardiography. European Society of Cardiology. Recommendations for chamber quantification. Eur J Echocardiogr 2006; 7: 79-108.

23. Devereux RB, Alonso DR, Lutas EM, et al. Echocardiographic assessment of left ventricular hypertrophy: comparison to necropsy findings. Am J Cardiol 1986; 57: 450-8.

24. Wierzbowska-Drabik K, Krzemińska-Pakuła M, Plewka M, et al. Relationship between echocardiographic parameters and exercise test duration in patients after myocardial infarction. Cardiol J 2009; 16: 507-13.

25. Wierzbowska-Drabik K, Krzemińska-Pakuła M, Chrzanowski L, et al. Age-dependency of classic and new parameters of diastolic function. Echocardiography 2008; 25: 149-55.

26. Cuspidi C, Lonati L, Sampieri L, et al. Impact of nocturnal fall in blood pressure on early cardiovascular changes in essential hypertension. J Hypertens 1999; 17: 1339-44.

27. Cuspidi C, Michev I, Meani S, et al. Non-dipper treated hypertensive patients do not have increased cardiac structural alterations. Cardiovasc Ultrasound 2003; 14: 1.

28. Cuspidi C, Lonati L, Sampieri L, et al. Impact of blood pressure control on prevalence of left ventricular hypertrophy in treated hypertensive patients. Cardiology 2000; 93: 149-54.

29. Cuspidi C, Macca G, Sampieri L, et al. Target organ damage and non-dipping pattern defined by two sessions of ambulatory blood pressure monitoring in recently diagnosed essential hypertensive patients. J Hypertens 2001; 19: 1539-45.

30. Stenehjem AE, Bjørnerheim R, Os I. From treatment to organ damage; a 5-year follow-up study of ambulatory blood pressure in essential hypertension. Diversity between development of left ventricular hypertrophy and urinary albumin excretion. Blood Press 2007; 16: 87-94.

31. Felício JS, Pacheco JT, Ferreira SR, et al. Hyperglycemia and nocturnal systolic blood pressure are associated with left ventricular hypertrophy and diastolic dysfunction in hypertensive diabetic patients. Cardiovasc Diabetol 2006; 5: 87-94.

32. Leitão CB, Canani LH, Kramer CK, et al. Blood pressure means rather than nocturnal dipping pattern are related to complications in type 2 diabetic patients. Diabet Med 2008; 25: 308-13.

33. Cicconetti P, Morelli S, De Serra C, et al. Left ventricular mass in dippers and nondippers with newly diagnosed hypertension. Angiology 2003; 54: 661-9.

34. Cuspidi C, Negri F, Muiesan ML, et al. Prevalence and severity of echocardiographic left ventricular hypertrophy in hypertensive patients in clinical practice. Blood Press 2010; $20: 3-9$.

35. Richey PA, Disessa TG, Somes GW, Alpert BS, Jones DP. Left ventricular geometry in children and adolescents with primary hypertension. Am J Hypertens 2010; 23: 24-9. 
36. Brady TM, Fivush B, Flynn JT, Parekh R. Ability of blood pressure to predict left ventricular hypertrophy in children with primary hypertension. J Pediatr 2008; 152: 73-8.

37. Grossman E, Alster Y, Shemesh J, Nussinovitch N, Rosenthal T. Left ventricular mass in hypertension: correlation with casual, exercise and ambulatory blood pressure. J Hum Hypertens 1994; 8: 741-6.

38. Pascual JM, Baldó E, Bertolín V, et al. Ambulatory arterial pressure and left ventricular hypertrophy in untreated hypertensive patients. Med Clin (Barc) 1999; 112: 166-170.

39. Feola M, Boffano GM, Procopio M, Reynaud S, Allemano P, Rizzi G. Ambulatory 24-hour blood pressure monitoring: correlation between blood pressure variability and left ventricular hypertrophy in untreated hypertensive patients. G Ital Cardiol 1998; 28: 38-44.

40. Hansen TW, Li Y, Boggia J, Thijs L, Richart T, Staessen JA. Predictive role of the nighttime blood pressure. Hypertension 2011; 57: 3-10.

41. Birkenhäger AM, van den Meiracker AH. Causes and consequences of a non-dipping blood pressure profile. Neth J Med 2007; 65: 127-31.

42. Cuspidi C, Meani S, Valerio C, et al. Body mass index, nocturnal fall in blood pressure and organ damage in untreated essential hypertensive patients. Blood Press Monit 2008; 13: 318-24.

43. Chamontin B, Amar J, Garelli-Flores II, Salvador M. Dippers and non-dippers among overweight hypertensive men. Blood Press Monit 1996; 1: 329-32.

44. Di Bello V, Talini E, Dell'Omo G, et al. Early left ventricular mechanics abnormalities in prehypertension: a twodimensional strain echocardiography study. Am J Hypertens 2010; 23: 405-12. 